\title{
Solution to the Twin Image Problem in Holography
}

\author{
Tatiana Latychevskaia and Hans-Werner Fink \\ Institute of Physics, University of Zurich, Winterthurerstrasse 190, CH-8057 Zurich, Switzerland \\ (Received 22 December 2006; revised manuscript received 11 March 2007; published 4 June 2007)
}

\begin{abstract}
While holography truly constitutes an ingenious concept, ever since its invention by Gabor it has been troubled by the so-called twin-image problem limiting the information that can be obtained from a holographic record. For symmetry reasons there are always two images appearing in the reconstruction of a hologram and the unwanted out of focus twin-image obscures the object. Here we show a universal method of reconstructing a hologram completely free of twin-image disturbances while no assumptions about absorbing or phase shifting properties of the object need to be imposed. Thus, truthful amplitude and phase distributions are retrieved.
\end{abstract}

DOI: 10.1103/PhysRevLett.98.233901

Scattering experiments aiming to obtain structural information about a microscopic object lack the ability to record the phase of the wave scattered by the object. Since detectors measure intensity distributions, the diffraction patterns contain information only about the amplitude of the scattered wave. However, for modern concepts of structural biology on the single molecule level, including recent developments around the free electron laser, solving the phase problem is a vital issue to ensure progress in this field. One way to detect the unknown phase has been available since the invention of the principle of holography $[1,2]$. By introducing a reference wave into the diffraction experiment, the phase of the object wave is directly measured by interference between object and reference wave, named as the hologram. More recently, an iterative numerical method to retrieve phase information from an oversampled diffraction pattern has been invented [3]. Whereas the oversampling method offers great promise, it does not record the phase directly, as in holography, but instead retrieves it iteratively from an initially as random assumed phase distribution.

While the beauty of holography is associated with directly delivered phase information and in principle achieving three-dimensional information of a microscopic object from just a single record, a major drawback is associated with the inherent unwanted twin image which obscures the reconstructed object.

To illustrate the situation, the basic setup for holography is depicted in Fig. 1 together with the positions of the object and its twin image when an inline hologram is reconstructed. In the object plane, the twin appears as an out of focus image while in the twin-image plane the object appears out of focus. The two images are mirror symmetric with respect to the point source. The problem of the twin images is especially pronounced for emission electron as well as for $\mathrm{x}$ - and gamma-ray [4,5] holography, where the source-object distances are small, and the reconstructed images of atoms are very close to their twin images from which they can hardly be distinguished. In some particular instances only, experimental efforts could remove the twin images [6-8]. In holography with visible light, the object
PACS numbers: 42.30.Rx, 42.25.Bs, 42.40.-i, 61.14.Nm

and its twin can be separated by using parallel beams and subtracting a second hologram from the reconstructed image [1,6] by employing a beam splitter [7] or introducing additional lenses into the recording and reconstructing scheme [8]. However, lenses are not available for x-ray or gamma-ray holography. In electron emission holography, the close proximity of source and sample also makes it impossible to employ lenses or a beam splitter between them. In other schemes, like holography with low energy electrons, lenses are to be avoided due to their inherent aberrations. Moreover, inline holography exhibits high phase sensitivity and is therefore, for coherent low energy electrons [9] and even for high energy electrons [10], for which DNA molecules represent extremely weak phase objects, the method of choice. The most widely employed approach to address the twin-image problem is to record a set of holograms at different wavelengths [11,12]. However, this method only suppresses but does not eliminate the twin image and is experimentally difficult to implement, in particular, when it comes to record fragile biological molecules subject to radiation damage. More recently, numerical methods to diminish the effect of the twin image have been proposed [13-18] but they are limited to purely absorbing objects ignoring phase shifts caused by the object, which is a coarse approximation of

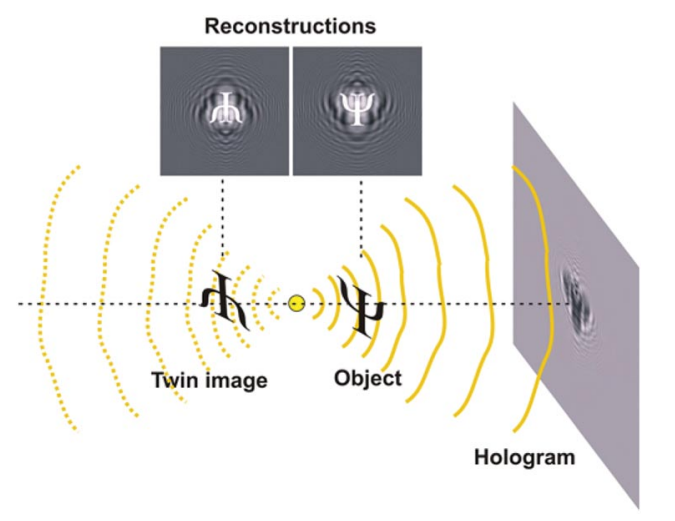

FIG. 1 (color). Position of the object and its twin image during hologram reconstruction. 
physical reality. Here we show how the twin image can be eliminated by numerical reconstruction of a hologram without imposing any restrictions on or assumptions about the object to be imaged.

A reference wave $A \exp (i k r)$, where $A$ is a complex constant and $\boldsymbol{r}$ is the radius vector to some point in space, propagates from a point source towards a distant screen illuminating it with the intensity $\left|A \exp \left(i k r_{s}\right)\right|^{2}=|A|^{2}=$ $B$, where $\boldsymbol{r}_{s}$ describes a point on the screen, thus providing a coherent background $B$. If an object is placed into the beam, the hologram is formed at the screen. It is brought about by interference between the wave scattered by the object and the unscattered wave. The transmission function in the object plane can be written as $\left[1-a\left(\boldsymbol{r}_{\text {obj }}\right)\right] \times$ $\exp \left[-i \varphi\left(\boldsymbol{r}_{\mathrm{obj}}\right)\right]$, where $a\left(\boldsymbol{r}_{\mathrm{obj}}\right)$ defines the absorbing properties of the object and $\varphi\left(r_{\text {obj }}\right)$ the phase shift introduced by the object with respect to the incident wave, and $\boldsymbol{r}_{\mathrm{obj}}$ describes a point of the object. On the other hand, the transmission function in the object plane can be written as $[1+$ $\left.t\left(\boldsymbol{r}_{\mathrm{obj}}\right)\right]$, where 1 corresponds to the transmittance in the absence of the object and $t\left(\boldsymbol{r}_{\mathrm{obj}}\right)$ is a complex function describing the presence of the object; both representations are equal:

$$
\left[1-a\left(\boldsymbol{r}_{\mathrm{obj}}\right)\right] \exp \left[-i \varphi\left(\boldsymbol{r}_{\mathrm{obj}}\right)\right]=1+t\left(\boldsymbol{r}_{\mathrm{obj}}\right) .
$$

Writing the transmission function as $\left[1+t\left(\boldsymbol{r}_{\mathrm{obj}}\right)\right]$ helps to identify the part of the incident beam which passes the object unscattered, thus forming the reference wave, $A \exp \left(i k r_{\text {obj }}\right)$. The part of the beam scattered by the object gives rise to the object wave, $A \exp \left(i k r_{\text {obj }}\right) t\left(\boldsymbol{r}_{\text {obj }}\right)$. The total field at the screen is the sum of the propagated reference and object waves $A\left[R\left(\boldsymbol{r}_{s}\right)+O\left(\boldsymbol{r}_{s}\right)\right]$, where $R\left(\boldsymbol{r}_{s}\right)=$ $\exp \left(i k r_{s}\right)$ and $O\left(\boldsymbol{r}_{s}\right)$ is the solution of the propagation integral [19]

$$
O\left(\boldsymbol{r}_{s}\right)=\iint t\left(\boldsymbol{r}_{\mathrm{obj}}\right) \exp \left(i k r_{\mathrm{obj}}\right) \exp \left(-i k \boldsymbol{r}_{\mathrm{obj}} \boldsymbol{r}_{s} / r_{s}\right) d \sigma_{\mathrm{obj}},
$$

where $\sigma_{\mathrm{obj}}$ denotes the object plane. The interference pattern on the screen yields a hologram with the transmission function $H\left(\boldsymbol{r}_{s}\right)=|A|^{2}\left|R\left(\boldsymbol{r}_{s}\right)+O\left(\boldsymbol{r}_{s}\right)\right|^{2}$. Dividing the hologram image by the background image results in $H\left(\boldsymbol{r}_{s}\right) / B\left(\boldsymbol{r}_{s}\right)=\left|R\left(\boldsymbol{r}_{s}\right)+O\left(\boldsymbol{r}_{s}\right)\right|^{2}$ which we call the normalized hologram. The background image shall be recorded with the exact same experimental conditions as the hologram, only in the absence of object, or be simulated from the hologram. It is worth noting that this normalized hologram is independent on $|A|^{2}$. It includes such factors as the point source intensity, and camera sensitivity. The following reconstruction routine can be applied to the normalized hologram without knowing the details of the data acquisition.

The final goal of our method is to reconstruct the distribution of the complex sum $\left[R\left(\boldsymbol{r}_{s}\right)+O\left(\boldsymbol{r}_{s}\right)\right]$. This is achieved by an iterative procedure [20] which basically boils down to the field propagation back and forth between screen and object plane until all artifacts due to the twin image are gone. It includes the following steps. (i) Formation of the input complex field as $\left[R\left(\boldsymbol{r}_{s}\right)+O\left(\boldsymbol{r}_{s}\right)\right]=$ $\left|R\left(\boldsymbol{r}_{s}\right)+O\left(\boldsymbol{r}_{s}\right)\right| \exp \left[i \Omega\left(\boldsymbol{r}_{s}\right)\right]$, where the amplitude is always given by the square root of the normalized hologram and the phase $\Omega\left(\boldsymbol{r}_{s}\right)$ is initially set to $k r_{s}$ - the phase of the known reference wave $R=\exp \left(i k r_{s}\right)$ - and it evolves towards its true value during iteration. (ii) Backpropagation to the object plane is simulated by solving the propagation integral [Eq. (2)]. (iii) The obtained complex field distribution in the object plane is multiplied with the conjugated incident wave $\exp \left(-i k r_{\text {obj }}\right)$ to extract the complex transmission function [1+t( $\left.\left.\boldsymbol{r}_{\mathrm{obj}}\right)\right]$, which provides the object's absorption $a\left(\boldsymbol{r}_{\mathrm{obj}}\right)$ and phase shift $\varphi\left(\boldsymbol{r}_{\mathrm{obj}}\right)$ according to Eq. (1). Thus, due to the presence of the reference wave, the correct absorption $a\left(\boldsymbol{r}_{\mathrm{obj}}\right)$ and phase properties $\varphi\left(\boldsymbol{r}_{\mathrm{obj}}\right)$ of the object can be extracted. The following constraint can be applied: $a\left(\boldsymbol{r}_{\mathrm{obj}}\right) \geq 0$, which refers to nothing else but the basic physical notion of energy conservation requiring that absorption may not lead to an increased amplitude following a scattering process. If negative values of absorption emerge, they are the result of the interference between twin image and reference wave, and they are subsequently replaced by zeros while the phase values remain unchanged. Based on this basic physical notion of positive absorption values, we obtain a constraint to derive a recombined absorption and phase distribution leading to the new transmission function for the object, by using Eq. (1):

$$
1+t^{\prime}\left(\boldsymbol{r}_{\mathrm{obj}}\right)=\left[1-a^{\prime}\left(\boldsymbol{r}_{\mathrm{obj}}\right)\right] \exp \left[-i \varphi\left(\boldsymbol{r}_{\mathrm{obj}}\right)\right] .
$$

(iv) Next, we let the reference wave $A \exp (i k r)$, originating from the point source, propagate forward. After it has passed the object with the new transmission function [1+ $\left.t^{\prime}\left(\boldsymbol{r}_{\text {obj }}\right)\right]$ it gives rise to the new complex sum $\left[R^{\prime}\left(\boldsymbol{r}_{s}\right)+\right.$ $\left.O^{\prime}\left(\boldsymbol{r}_{s}\right)\right]$ in the screen plane. We then acquire the altered phase value from this new sum and use it as the input phase value for the next iteration starting at step (i). Already the first iteration reconstructs the complex object with the same quality as the conventional hologram reconstruction routines. Further iterations eventually lead to the elimination of the twin term. There are no limitations on the object's size or its properties: it can be a weak or strong absorbing and/or a weak or strong phase shifting object. Our method also does not require a certain object's surrounding or so-called support, as most known iterative methods do [21,22]. The object and its surrounding can be anything: for instance, an extended biological molecule which is identified only by a weak phase shift of the incident beam or an arrangement of heavy atoms acting as pointlike absorbing centers. The following discussion is limited to the approximation of single scattering events.

We now test the routine, first with a simulated hologram, then by using experimental holograms. A hologram of an extended object with a maximum absorption $a\left(\boldsymbol{r}_{\mathrm{obj}}\right)$ of $80 \%$ of an incident beam and a maximum phase shift $\varphi\left(r_{\text {obj }}\right)$ of 
(a)

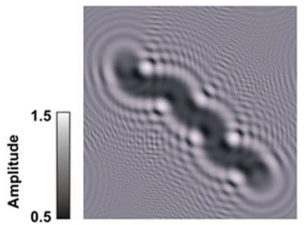

(b)
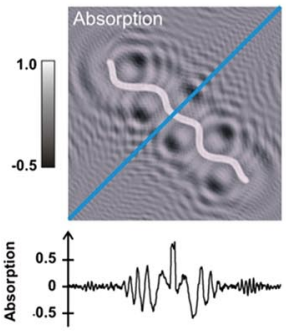

(c)

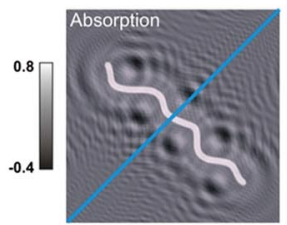

(d)
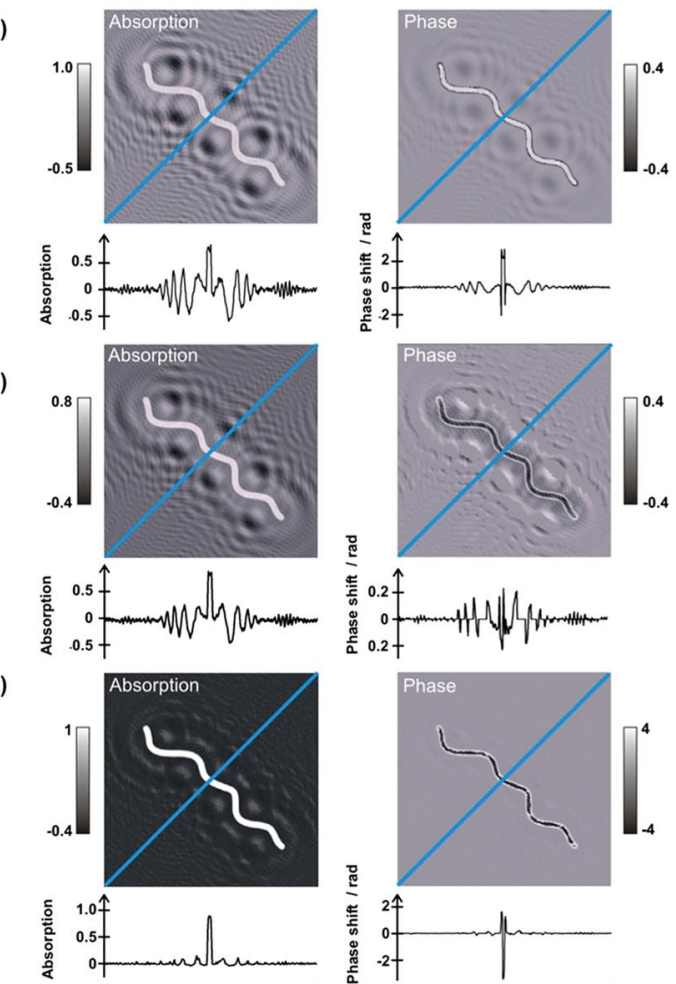

(e)
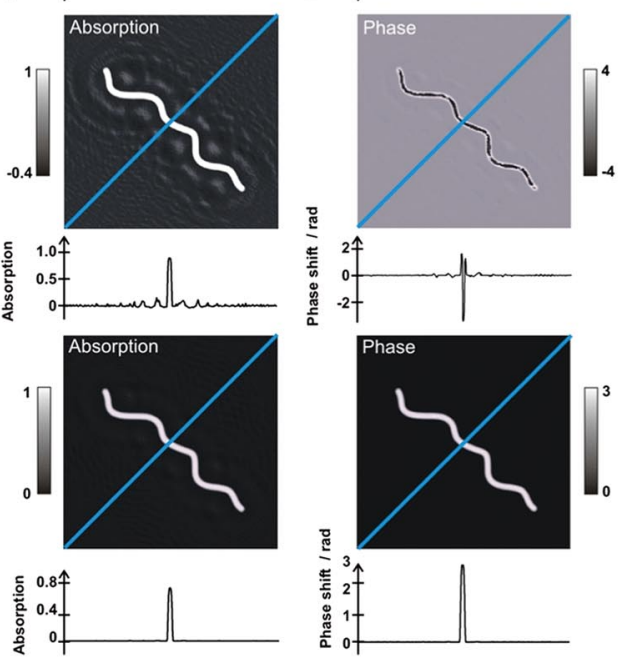

FIG. 2 (color). Simulated and reconstructed hologram of an extended object. (a) Normalized hologram. (b) Reconstructed amplitude and phase by conventional reconstruction. (c) Reconstructions after the first iteration. (d) Reconstructions after the tenth iteration. (e) Reconstructions after the fiftieth iteration. Below each reconstruction the intensity distributions along the blue lines are displayed. The error of the reconstruction was estimated as the mean squared error $E=\frac{1}{N^{2}} \sum_{\substack{i=1, N \\ j=1, N}} \mid \rho(i, j)-$ $\left.\rho_{0}(i, j)\right|^{2}$, where $\rho(i, j)$ is the reconstructed distribution, $\rho_{0}(i, j)$ is the initial distribution, and $N$ is the number of pixels. The errors of the reconstructions after 50 iterations, defined in the above way, are $E=1.4 \times 10^{-7}$ for the absorption distribution and $E=4.6 \times 10^{-7}$ for the phase distribution.

3 radians was simulated by using the propagation integral and reconstructed with our method described above. The results are shown in Fig. 2. The object's absorption and phase distributions obtained by the conventional hologram reconstruction routine are shown in Fig. 2(b) and those obtained after the first iteration in Fig. 2(c). Both recon- structions are similar and suffer from the superimposed out of focus twin image. While the reconstructed absorption distributions are almost identical, the phase distribution obtained by the conventional reconstruction is initially better since it shows a maximum phase shift of 2.5 radians [see Fig. 2(b)]. Nevertheless, it does not reach the correct predefined maximum phase shift of 3 radians. Each further iteration step causes the twin image to fade away in the reconstructed absorption distribution while the phase distribution approaches its true value. After the first iteration, the maximal phase shift amounts to only 0.2 radians [see Fig. 2(c)]. However, already after the tenth iteration [see Fig. 2(d)] the phase distribution appears almost free from the disturbing twin image and the phase values are recovering. The absorption and phase distributions, retrieved after the fiftieth iteration, shown in Fig. 2(e), demonstrate that the effect of the twin image has been completely re-

(a)

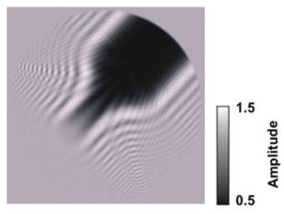

(b)
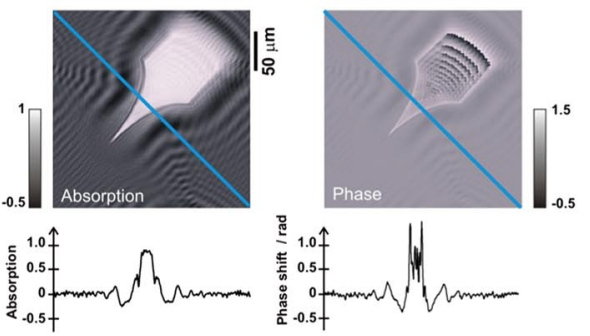

(c)
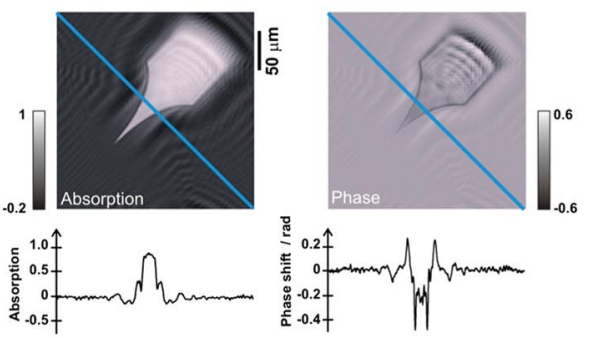

(d)
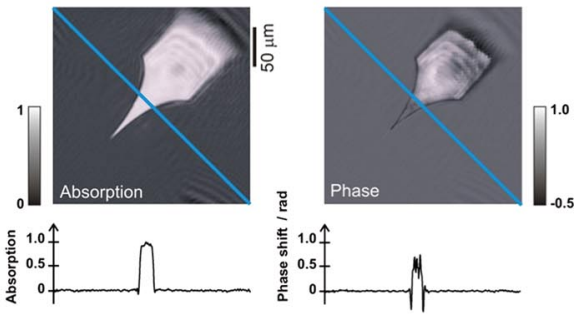

FIG. 3 (color). Iteratively reconstructed experimental optical hologram of a tungsten tip. (a) Normalized hologram. (b) Reconstructed absorption and phase distributions by conventional reconstruction. (c) Reconstructions after the first iteration; oscillations due to the twin image are apparent. (d) Reconstructions after the 500th iteration. Below each reconstruction the intensity distributions along the blue lines are displayed. A cosine window filter was applied to the hologram to avoid artifacts from edges while Fourier transforming. 
(a)

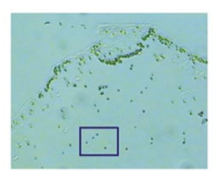

(b)

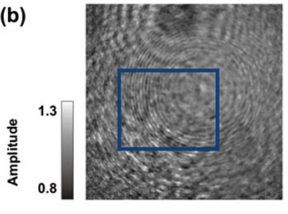

(c)

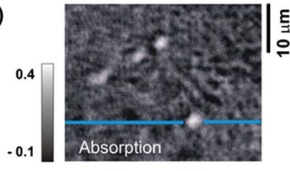

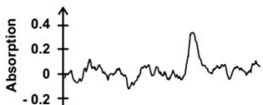

(d)
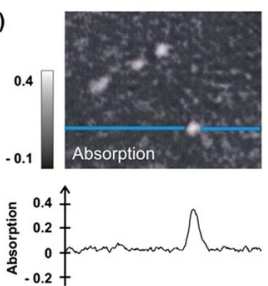
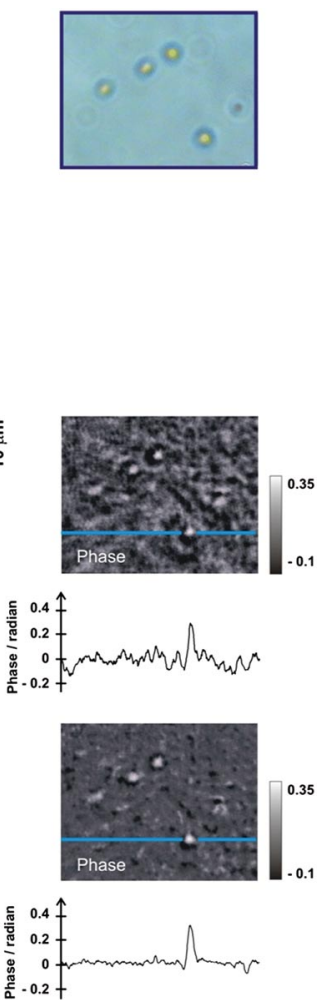

FIG. 4 (color). Iteratively reconstructed experimental optical hologram of latex spheres of $1 \mu \mathrm{m}$ in diameter. (a) Conventional optical micrographs of latex spheres deposited onto a glass coverslip. The area marked in blue and magnified at the right was subject to the divergent laser beam to generate the holograms. (b) Normalized hologram. (c) Reconstructed absorption and phase distributions by conventional reconstruction. (d) Reconstructions after the 1000th iteration. Below each reconstruction the intensity distributions along the blue lines are displayed.

moved and the phase has finally reached its predefined value of 3 radians. This demonstrates that the twin-image removal is more than just getting rid of an artifact of holography; it is also a way to arrive at the true phase values.

To apply our method to experimental holograms, green laser light of $532 \mathrm{~nm}$ wavelength is focused by means of a microscope objective. At the focal point, a pinhole of $10 \mu \mathrm{m}$ in diameter optimizes the beam to form a coherent point source. A tungsten tip is then placed into the divergent beam and controlled by a $x y z$-movable stage. The distance between the point source and the tip is adjusted to approximately $0.8 \mathrm{~mm}$. A screen is placed at about $1 \mathrm{~m}$ from the point source and the hologram captured by a CCD camera. The recorded and normalized hologram and its reconstructions are shown in Fig. 3. A tungsten tip is a strongly absorbing object and it is apparent that about $1 / 4$ of the recorded hologram must be attributed to the strong object wave. This, in turn, demonstrates that our method does not require the traditional holography postulate of a strong and dominating reference wave. The result after 500 iterations shows that residues due to the twin image in the

reconstructed absorption and phase distributions are gone. We also evaluated our method by experimentally realizing the other extreme situation of weak scattering microscopic objects. For this we used $1 \mu \mathrm{m}$ in diameter latex spheres, deposited onto a glass coverslip, and placed at about $0.2 \mathrm{~mm}$ from pinhole. The hologram was recorded at the screen, placed about $7 \mathrm{~cm}$ away from the point source. The normalized hologram as well as its traditional reconstruction and the application of our iterative method are shown in Fig. 4.

With this, a novel method to finally solve the twin-image problem is established and can now be applied without limitations to wavelength or wave front shapes (planar or spherical), for imaging objects of arbitrary size, exhibiting absorbing and/or phase shifting properties. From a single holographic record, twin-image free true absorption and phase distributions are iteratively retrieved.

We would like to thank Conrad Escher for his help and for carefully reading the manuscript. The work presented here is supported by the European Project SIBMAR.

[1] D. Gabor, Nature (London) 161, 777 (1948).

[2] D. Gabor, Proc. R. Soc. A 197, 454 (1949).

[3] J. Miao, D. Sayre, and H. N. Chapman, J. Opt. Soc. Am. A 15, 1662 (1998).

[4] M. Tegze and J. Faigel, Nature (London) 380, 49 (1996).

[5] P. Korecki and J. Korecki, Hyperfine Interact. 144, 85 (2002).

[6] W. L. Bragg and G. L. Roger, Nature (London) 167, 190 (1951).

[7] E. Leith and J. Upatnieks, J. Opt. Soc. Am. 53, 1377 (1963).

[8] O. Bryngdah and A. Lohmann, J. Opt. Soc. Am. 58, 620 (1968).

[9] H.-W. Fink, H. Schmid, E. Ermantraut, and T. Schulz, J. Opt. Soc. Am. A 14, 2168 (1997).

[10] T. Matsumoto, T. Tanji, and A. Tonomura, Optik (Jena) 100, 71 (1995).

[11] S. Y. Tong, H. Hua Li, and H. Huang, Phys. Rev. Lett. 67, 3102 (1991).

[12] J. J. Barton, Phys. Rev. Lett. 67, 3106 (1991).

[13] K. A. Nugent, Opt. Commun. 78, 293 (1990).

[14] G. Liu and P. D. Scott, J. Opt. Soc. Am. 4, 159 (1987).

[15] L. Onural and P. D. Scott, Opt. Eng. (Bellingham, Wash.) 28, 1124 (1987).

[16] X. M. H. Huang, J. M. Zuo, and J.C.H. Spence, Appl. Surf. Sci. 148, 229 (1999).

[17] J.F. Arocena, T.A. Rotwell, and M.R. A. Shegelski, Micron 36, 23 (2005).

[18] A. L. Bleloch, A. Howie, and E. M. James, Appl. Surf. Sci. 111, 180 (1997).

[19] J. J. Barton, Phys. Rev. Lett. 61, 1356 (1988).

[20] R. W. Gerchberg and W. O. Saxton, Optik (Jena) 35, 237 (1972).

[21] J. R. Fienup, Opt. Lett. 3, 27 (1978).

[22] J. R. Fienup, Appl. Opt. 21, 2758 (1982). 